\title{
MICROGRAVITY SEGREGATION IN COLLISIONAL GRANULAR SHEARING FLOWS
}

\author{
M.Y. LOUGE, J.T. JENKINS, A. REEVES AND S. KEAST \\ Cornell University \\ Upson Hall, Ithaca, NY 14853
}

\begin{abstract}
This article concerns granular shearing flows in microgravity where segregation of grains by size or mass is driven by spatial gradients in the fluctuation energy of the grains.

Experiments were conducted on NASA's microgravity aircraft with a shear cell shaped like a race track and containing a mixture of two types of spherical grains. A gradient of fluctuation energy was produced between the inner, energetic moving boundary driving collisions among the grains and the outer, more dissipative boundary at the periphery of the cell. The energetics were achieved by covering the two boundaries with bumps of appropriate size and spacing and by setting the distance between the flat side walls through which the flow was observed.

The grain segregation and the corresponding velocity statistics were captured by a high-speed video camera and analyzed using computer vision software. The resulting profiles of particle mean and fluctuation velocities were compared with computer simulations of the entire experimental apparatus.
\end{abstract}

\section{Introduction}

The size segregation of flowing or shaken grains is a commonly observed phenomenon in industrial processes and in nature. In systems that do not involve much agitation of the grains, segregation mechanisms involving gravity include the preferential downward percolation of smaller particles in relatively slow inclined shear flows [1], the upward frictional ratchetting of large particles [2], and the preferential filling of space beneath larger particles by smaller particles in a system that is occasionally shaken $[3,4]$. In sheared or vibrated systems of colliding grains, gravity also influences mixtures of grains of different sizes. Here, buoyant forces act to separate grains that differ in size and, consequently, in the local volume that they displace.

In reduced gravity, buoyancy is suppressed and attention can be focused on another segregation mechanism that involves highly agitated flows and is driven by spatial gradients in the energy of granular velocity fluctuations. In steady, fully-developed flows, the balance of momentum exchanged in collisional interaction among different species of grains requires that concentration gradients be balanced by gradients of particle 


\section{M.Y. LOUGE, J.T. JENKINS, A. REEVES AND S. KEAST}

fluctuation energy. Reduced gravity also eliminates the possibility that a collisional flow may condense into a slower, denser flow dominated by enduring contacts.

Because collisions inevitably dissipate energy, collisional granular shear flows have limited extent in the direction transverse to the flow and, consequently, are strongly influenced by their boundaries. Because grains, on average, slip relative to the latter, a bumpy or frictional boundary can locally convert slip energy into fluctuation energy. This production competes with the dissipation of fluctuation energy arising in collisions with the boundary. A similar competition between the working of the mean shear and collisional dissipation largely determines the level of fluctuation energy in the interior. Although boundaries with a large slip velocity tend to create high local levels of fluctuation energy, they also promote smaller mean velocities in the interior, which in turn limit the magnitude of the fluctuation kinetic energy there.

In a shearing flow bounded by two bumpy walls normal to the gradient and two flat walls normal to the mean vorticity, the size and spacing of the bumps and the distance between the flat walls can be designed to adjust the granular slip velocity and the rates of production or dissipation of fluctuation energy [5]. This permits the control of the component of the spatial gradient of the fluctuation energy that is perpendicular to the flow. Such gradients in fluctuation energy may be exploited to drive the separation by size or other properties in a binary mixture of spherical grains.

To observe this phenomenon, we have constructed a shear cell in the form of a race track in which the segregation of a sheared binary mixture of two different types of sphere is maintained by the motion of the inner boundary relative to the outer. The experiments are meant to isolate two different sub-mechanisms of collisional segregation that usually occur together: the first is associated with differences in the inertia of the grains; it is achieved with spheres of different masses, but equal diameters. The second is associated with differences in the geometry of the grains; we approximate it using a mixture of grains of different diameter and densities.

We conducted experiments with a prototype shear cell on NASA's KC-135 microgravity aircraft. Because in the absence of gravity the only available time scale is proportional to the speed of the moving boundary, we could reduce that speed and avoid particle damage without affecting the phenomenon under study. The relatively low speed also allowed us to track the grain motion with a digital video camera.

We developed a computer vision software to analyze the resulting images and compared the profiles of volume fraction and mean and fluctuating velocities with computer simulations of the apparatus. The link between the computer simulations and the physical experiments was achieved by measuring in our laboratory the parameters that characterize individual impacts [6,7].

We begin with a description of the computer simulations and the apparatus. We then report experimental results and compare those with predictions of the simulations.

\section{Simulations}

Computer simulations guided our design of the microgravity shear cell and informed the development of theory. In particular, they prescribed a cell geometry featuring regions 


\section{MICROGRAVITY SEGREGATION}

of nearly fully-developed flow and showed that measurements through the side walls closely represented the state of flow in the interior.

The simulations follow the dynamics of an ensemble of two species of spheres of diameters $\sigma_{1}$ and $\sigma_{2}$ and material densities $\rho_{1}$ and $\rho_{2}$ interacting with the boundariesand among themselves through individual impacts. They are based on the algorithm described by Hopkins and Louge [8]. In that algorithm, collisions occur when a sphere overlaps slightly with another sphere or with the wall. The algorithm adjusts its time step periodically to ensure that the mean overlap is kept below a negligible tolerance. In addition, a search grid is superimposed on the flow domain to permit fast identification of near neighbors. Because this method makes it superfluous to maintain a list of future impacts, its computing time is merely proportional to the number of spheres and, consequently, it can simulate the entire shear cell on a relatively small workstation. Figure 1 shows a simulated microgravity shear cell of size modest enough to distinguish details of the flow.

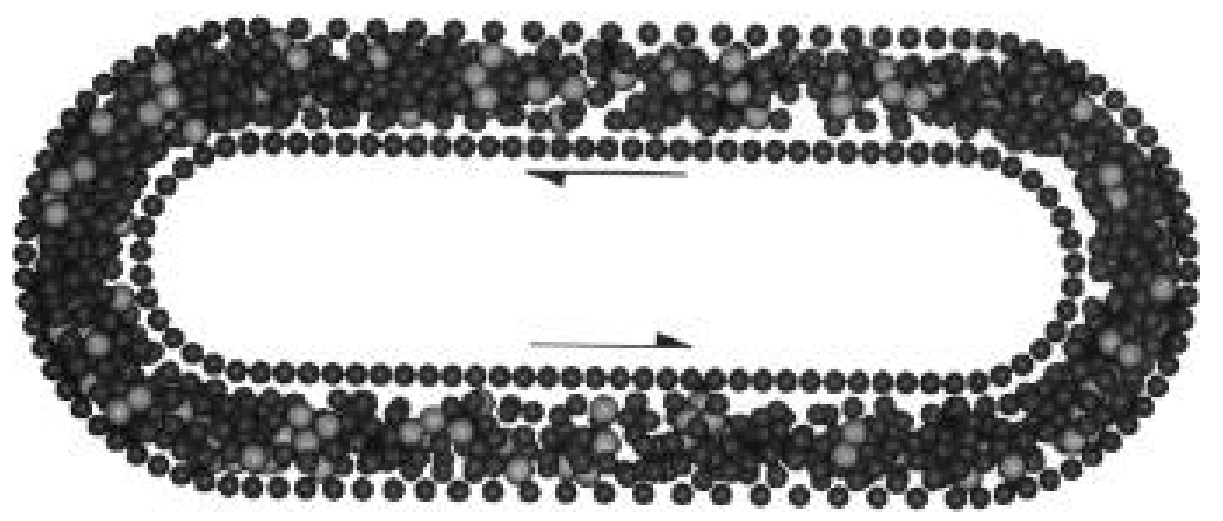

Figure 1. A shear cell of modest size with two species that differ in diameter. The outer boundary is fixed. The inner boundary moves in the direction shown.

Profiles of solid volume fraction, velocity and temperature for the two grain species were measured by dividing the flow domain into a number of averaging slices (Fig. 2). The average value $\langle\psi\rangle$ of an intrinsic grain property $\psi$ in a slice was calculated by considering a number $\mathbf{J}$ of instantaneous realizations of the flow, by summing all contributions from $\mathrm{K}$ spheres passing through the slice over all realizations, and by dividing the result by $\mathrm{J}$ and $\mathrm{K}$. This center-averaging is consistent with the kinetic theory. Because the flow could contain two species of different masses, the second moments of velocity fluctuations were mass-weighted. For the second moment of species $\mathrm{j}$ along direction $\mathrm{i}$ :

$$
\mathrm{T}_{\mathrm{i}}(\mathrm{j}) \equiv\left\langle\mathrm{m}(\mathrm{j})\left(\mathrm{u}_{\mathrm{i}}(\mathrm{j})-\left\langle\mathrm{u}_{\mathrm{i}}(\mathrm{j})\right\rangle\right)^{2}\right\rangle,
$$

where $m(j)$ is the mass of a species $j$ sphere and $u_{i}(j)$ is its velocity in the direction $i$. The temperature of species $j$ is then $T(j)=\left[T_{1}(j)+T_{2}(j)+T_{3}(j)\right] / 3$. In the absence of gravity, all grain velocities scaled with the velocity $U$ of the boundary. In addition, we 


\section{M.Y. LOUGE, J.T. JENKINS, A. REEVES AND S. KEAST}

made the second moments in Eq. (1) dimensionless with $\mathrm{U}^{2}$ and the mass of small spheres. Dimensionless quantities are denoted by a cross.

Because any rigid boundary tends to order spheres in its neighborhood, the transverse profiles of solid volume fraction exhibit spatial oscillations near the bumpy inner and outer walls with wavelength of order a sphere diameter. Because the kinetic theory ignores these fluctuations, their presence can hinder comparisons of the measured segregation with the corresponding theoretical predictions. In grain mixtures, we alleviate this difficulty by focusing on the relative number fraction $\phi$ rather than the volume fractions of each individual species. For example, for species 1,

$$
\phi_{1}=\frac{\left(\sigma_{2} / \sigma_{1}\right)^{3}+\bar{v}_{2} / \bar{v}_{1}}{\left(\sigma_{2} / \sigma_{1}\right)^{3}+v_{2} / v_{1}} \text {. }
$$

where the overbar denotes averaging in the transverse direction. Values of $\phi_{1}$ above unity indicate a local surplus of species 1 spheres. As long as $\sigma_{1}$ and $\sigma_{2}$ are not greatly different, the relative number fractions incorporate ratios of volume fractions that nearly oscillate in phase and, consequently, their own fluctuations are considerably reduced.

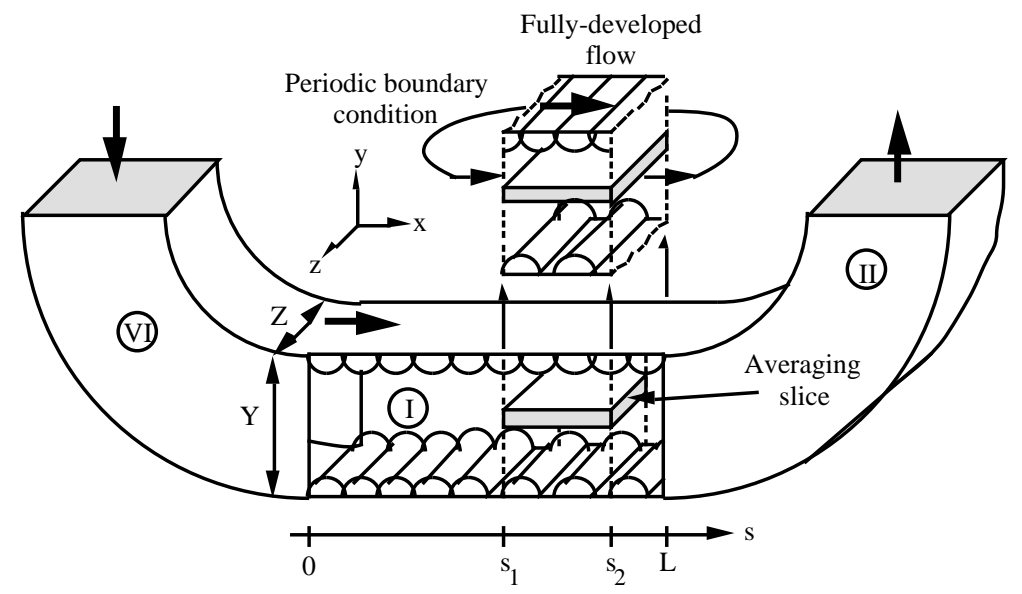

Figure 2. Sketch of the straight section (I) of the simulations with length L, width Y between centers of the cylindrical boundary bumps shown and depth $\mathrm{Z}$ between the two flat side walls. Bumps are affixed to both boundaries, including the curved regions (II) and (IV) and the upper straight section (III) that is omitted for clarity. Fully-developed flows are simulated using a periodic boundary condition in the "observation region" with $\mathrm{s}_{1} \leq \mathrm{s} \leq \mathrm{s}_{2}$. Dimensions are not to-scale.

In the simulations, impacts were characterized in terms of three coefficients [9]. The first is the coefficient of normal restitution e. The second is the coefficient of friction $\mu$ for sliding impacts. The last is the coefficient of tangential restitution $\beta_{0}$ for impacts that do not involve sliding. For collisions between two free spheres or between a sphere and one of the flat side walls, we measured these parameters with the facility described by Foerster et al [7]; for collisions with a cylindrical boundary bump, we adopted the method of Lorenz et al [6]. Table 1 summarizes the results. 


\section{MICROGRAVITY SEGREGATION}

Table 1 - Impact properties

\begin{tabular}{|c|c|c|c|c|c|c|c|c|}
\hline $\begin{array}{c}\text { sphere } \\
1\end{array}$ & $\begin{array}{c}\sigma_{1} \\
(\mathrm{~mm})\end{array}$ & $\begin{array}{c}\rho_{1} \\
\left(\mathrm{~g} / \mathrm{cm}^{3}\right)\end{array}$ & $\begin{array}{c}\text { sphere } 2, \\
\text { bump or wall }\end{array}$ & $\begin{array}{c}\sigma_{2} \\
(\mathrm{~mm})\end{array}$ & $\begin{array}{c}\rho_{2} \\
\left(\mathrm{~g} / \mathrm{cm}^{3}\right)\end{array}$ & $\mathrm{e}$ & $\mu$ & $\beta_{0}$ \\
\hline \hline acrylic & $3.2 / 3.96$ & 1.22 & acrylic & $3.2 / 3.96$ & 1.22 & 0.93 & 0.12 & 0.35 \\
\hline ceramic & 3.18 & 3.86 & ceramic & 3.18 & 3.86 & 0.97 & 0.10 & 0.24 \\
\hline acrylic & $3.2 / 3.96$ & 1.22 & ceramic & 3.18 & 3.86 & 0.93 & 0.11 & 0.10 \\
\hline acrylic & $3.2 / 3.96$ & 1.22 & fixed bump & 3.18 & - & 0.97 & 0.22 & 0.28 \\
\hline ceramic & 3.18 & 3.86 & fixed bump & 3.18 & - & 0.68 & 0.08 & 0.29 \\
\hline acrylic & $3.2 / 3.96$ & 1.22 & aluminum & $\infty$ & - & 0.94 & 0.14 & 0.51 \\
\hline ceramic & 3.18 & 3.86 & aluminum & $\infty$ & - & 0.61 & 0.10 & 0.14 \\
\hline acrylic & $3.2 / 3.96$ & 1.22 & glass & $\infty$ & - & 0.83 & 0.12 & 0.34 \\
\hline ceramic & 3.18 & 3.86 & glass & $\infty$ & - & 0.96 & 0.09 & 0.00 \\
\hline
\end{tabular}

\section{Shear Cell}

With insight from the simulations, we designed the shear cell to visualize steady, fullydeveloped granular segregation in a rectilinear shearing flow without significant body forces. The cell has dimensions $\mathrm{L}=419 \mathrm{~mm}, \mathrm{Y}=29 \mathrm{~mm}, \mathrm{Z}=40 \mathrm{~mm}$, and its moving boundary has a radius $R=62 \mathrm{~mm}$.

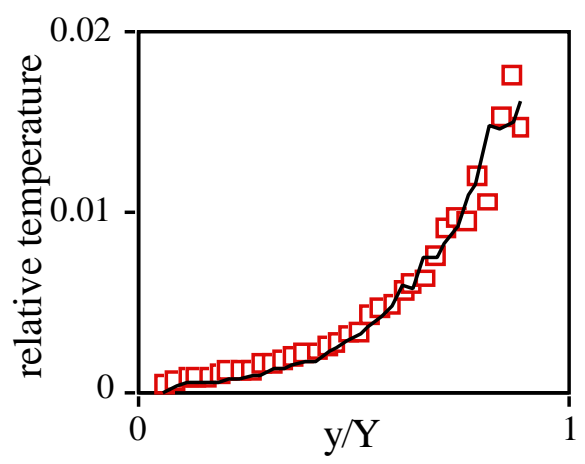

Figure 3. Comparison of simulated transverse profiles of the dimensionless granular temperature of ceramic spheres calculated from the observation region of the camera (line) and from a similar fully-developed region with periodic boundaries (symbols) with conditions of test $\mathrm{A}$. The abscissa is the relative distance from the outer bumpy boundary.

Flat side walls with windows provided lateral containment and permitted the flow to be viewed. A digital Kodak EktaPro R0 camera imaged a $25 \mathrm{~mm}$ wide region of the cell in the range $292 \leq \mathrm{s} \leq 326 \mathrm{~mm}$ at a frame rate $\mathrm{F}$ of 500 or $1000 \mathrm{~Hz}$. To verify that the flow observed with the camera was nearly fully-developed, we compared transverse profiles of mean and fluctuation velocities obtained in the observation region 


\section{M.Y. LOUGE, J.T. JENKINS, A. REEVES AND S. KEAST}

from simulations of the entire cell against identical simulations with periodic boundaries in the direction normal to the flow (Fig. 3).

The inner boundary consists of a specialty chain to which stainless steel segments of $9.5 \mathrm{~mm}$ width are attached. Three closely spaced hemi-cylindrical stainless steel rods of $3.2 \mathrm{~mm}$ diameter perpendicular to the flow direction were vacuum-brazed onto these segments to roughen the moving boundary. The chain is driven by dynamicallybalanced sprockets that produce negligible vibrations. It was run without lubrication to prevent any corruption of the frictional impact coefficients of the interior grains and the bumps. In the linear section of the cell, plastic rubbing strips support the chain while stabilizing its motion and avoiding artificially low restitution coefficients in its impacts with interior grains. The surface of the outer stationary boundary is also made bumpy with hemi-cylinders of $3.2 \mathrm{~mm}$ perpendicular to the flow direction and with centers separated by $4.8 \mathrm{~mm}$.

The chain is driven by a dc motor capable of delivering a torque of $2 \mathrm{~N} . \mathrm{m}$ at 700 RPM and connected to one of the sprockets through a timing belt. The motor is controlled through a servo amplifier receiving angular velocity feedback from a magnetic tachometer mounted on the other sprocket. On the aircraft, the z-direction perpendicular to the flat walls was aligned with the principal direction of gravitational accelerations.

\section{Experiments}

Table 2 summarizes experimental conditions for the flight tests in the $\mathrm{KC}-135$ microgravity aircraft. The symbols $\bar{v}_{1}$ and $\bar{v}_{2}$ represent the fractions of the volume of the whole cell occupied by species 1 and 2, respectively. At these levels of overall packing, the simulations indicated that these mean volume fractions were nearly identical to those in the viewing regions.

$\underline{\text { Table } 2 \text { - Experimental conditions }}$

\begin{tabular}{|c|c|c|c|c|c|c|c|c|c|c|c|c|}
\hline Test & $\begin{array}{c}\mathrm{U} \\
\mathrm{m} / \mathrm{s}\end{array}$ & $\begin{array}{c}\mathrm{F} \\
\mathrm{Hz}\end{array}$ & Spec. 1 & $\begin{array}{c}\rho_{1} \\
\mathrm{~g} / \mathrm{cm}^{3}\end{array}$ & $\begin{array}{c}\sigma_{1} \\
\mathrm{~mm}\end{array}$ & $\begin{array}{c}\bar{v}_{1} \\
\%\end{array}$ & Spec. 2 & $\begin{array}{c}\rho_{2} \\
\mathrm{~g} / \mathrm{cm}^{3}\end{array}$ & $\begin{array}{c}\sigma_{2} \\
\mathrm{~mm}\end{array}$ & $\begin{array}{c}\overline{\mathrm{v}}_{2} \\
\%\end{array}$ & $\mathrm{t}^{\dagger}$ & $\begin{array}{c}\tau \\
\mathrm{s}\end{array}$ \\
\hline $\mathrm{A}$ & 1.4 & 1000 & ceramic & 3.86 & 3.18 & 10 & acrylic & 1.22 & 3.96 & 30 & 300 & 10 \\
\hline $\mathrm{B}$ & 1.4 & 1000 & acrylic & 1.22 & 3.2 & 20 & ceramic & 3.86 & 3.18 & 20 & 130 & 10 \\
\hline $\mathrm{C}$ & 0.7 & 500 & acrylic & 1.22 & 3.2 & 31 & acrylic & 1.22 & 3.96 & 10 & 500 & 15 \\
\hline
\end{tabular}

The spheres were selected from many materials for the relatively small collisional energy dissipation that their low friction and high restitution produced. They also exhibited a finish that allowed the vision software to track their movement with accuracy. We retained Engineering Laboratories and Hoover Precision to manufacture, respectively, colored acrylic and ceramic grains of excellent sphericity $(\sigma \pm<0.3 \%)$ and narrow size distribution $(\sigma \pm<0.2 \%)$. Electrostatic charging of these dielectric solids was mitigated by maintaining high relative humidity in the apparatus. With this precaution, no evidence of such charging could be observed. 


\section{MICROGRAVITY SEGREGATION}

A typical KC-135 parabola included a gravitational pull of order $18 \mathrm{~m} / \mathrm{s}^{2}$ followed by 20 seconds of reduced gravity. A tri-axial accelerometer indicated that residual gravitational accelerations in the aircraft did not exceed $\pm 0.1 \mathrm{~m}^{2} / \mathrm{s}$. We began each test by starting the motor at the onset of reduced gravity.

The computer simulations helped us prescribe mixtures for which a constant residual gravitational acceleration $\mathrm{g}^{\dagger}=\mathrm{g}_{\mathrm{z}} \sigma_{1} / \mathrm{U}^{2}$ in the $\mathrm{z}$-direction would produce minimum average sinking of the most massive spheres. The worst case involved ceramic spheres in Test A. There, with a residual acceleration $\mathrm{g}^{\dagger}=0.00063$, on the order the observed peak-to-peak fluctuations, the simulations predicted a sinking distance of merely $1.5 \sigma_{1}$ without any apparent change in the granular temperature profile.

Using the same simulations, we also estimated the dimensionless time $\mathrm{t}^{\dagger}=\mathrm{t} \mathrm{U} / \mathrm{Y}$ required for each experiment to re-establish steady segregation after canceling that level of residual acceleration. Based on that estimate, we waited a time $\tau$ after the onset of reduced gravity to acquire images with the camera (Table 2 ).

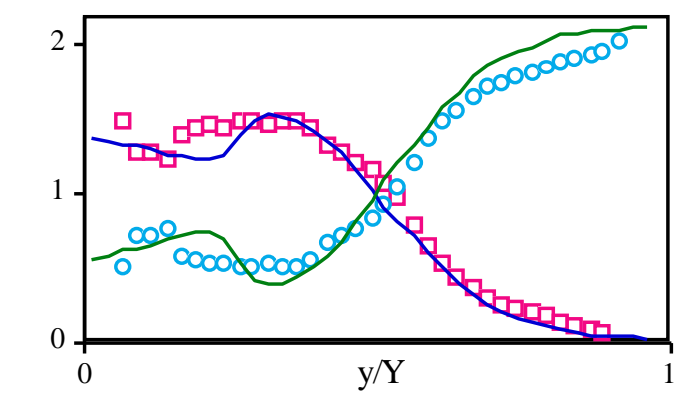

Figure 4. Comparison of simulated transverse profiles of $\phi$ estimated from area fractions observed through the side window (lines) and calculated from profiles of interior volume fraction averaged in the z-direction (symbols) for the conditions of Test B. The squares and circles represent ceramic and acrylic spheres, respectively.

The simulations also helped develop a strategy for relating the surface fractions of the two species observed through the side walls to the corresponding fractional populations in the interior. Generally, the vision algorithm detected grains that were within a sphere diameter from the window. The two species were then distinguished according to their distinct gray scale or size. Each frame was subdivided into ten horizontal strips of constant width. Strips spanned the entire length of the image to reflect the fully-developed state of the flow. By merely incrementing the observed sphere cross-sectional area intersecting each strip, the vision algorithm calculated the fraction $\alpha$ of the strip surface occupied by each species within the field of view and estimated the corresponding relative number fraction by substituting $\alpha$ for $v$ in Eq. (2). We tested this measurement strategy using computer simulations. As Fig. 4 illustrates, the simulated transverse profiles of $\phi$ produced by this method closely represent the state of segregation in the interior of the simulated flow.

We developed a computer image tracking analysis for this application. It consisted of five principal steps. In the first, a gradient image was computed with the Sobel edge 


\section{M.Y. LOUGE, J.T. JENKINS, A. REEVES AND S. KEAST}

operator from the initial digitized gray-scale image. The resulting edges were then thinned by retaining only the pixel of largest intensity in the gradient direction. Next, a binary image was generated by setting to one the intensity of pixels above a certain threshold. The result was correlated with a thin binary circle of diameter equal to that of the species of interest. Finally, a hill-climbing procedure reached the sphere centers at the peaks of the resulting correlation field. In subsequent images, the algorithm repeated those steps only in small regions adjacent to the detected centers.

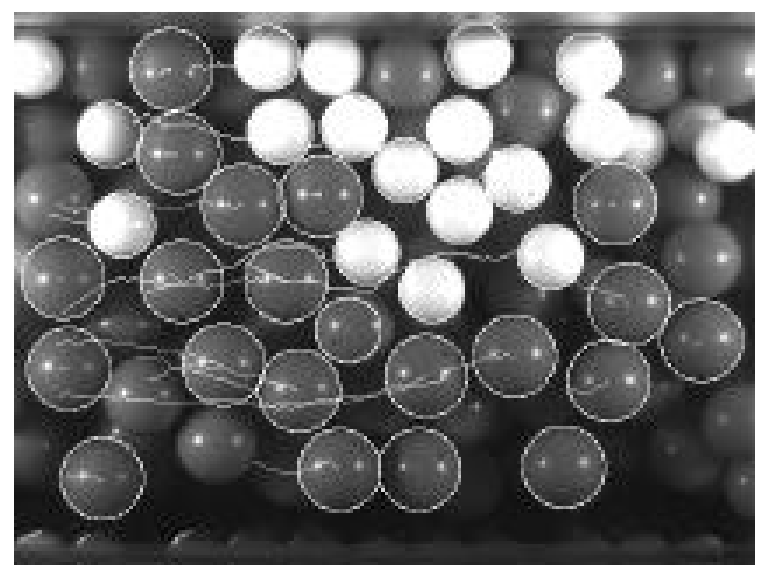

Figure 5. A typical image for the conditions of Test A. Circles and lines are superimposed to indicate the location and trajectory of detected spheres. The moving boundary can be discerned at the bottom of the picture.

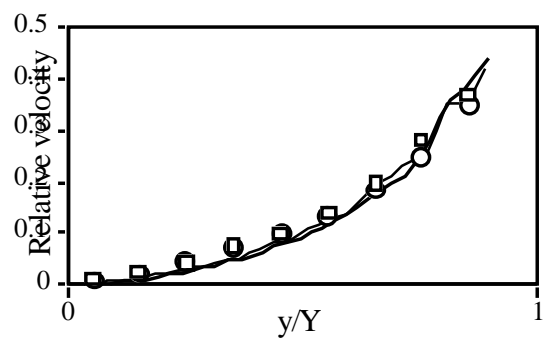

Figure 6. Transverse profiles of mean velocity relative to the chain speed for the conditions of Test A. The symbols and lines are experimental measurements and predictions of the simulations, respectively. The circles and thin line are for acrylic spheres; the squares and thick line for ceramic.

As long as the displacement between two consecutive frames remained less than a sphere radius, this method permitted unambiguous tracking of the moving spheres. Periodically, as the original spheres progressively left the field of view, the software initiated a new search in the entire image. Adequate lighting proved to be an important condition for success of the algorithm. The field of view was illuminated with two optical fiber light guides. The shutter speed of the camera, the opening of the lens and the illumination level were tuned by trial and error until grains could be reliably detected within a sphere diameter from the window. 


\section{MICROGRAVITY SEGREGATION}

Figure 5 is a typical image from the digital camera. Velocities were calculated from the positions of each sphere center in two consecutive images and the corresponding strip statistics were incremented as outlined earlier.

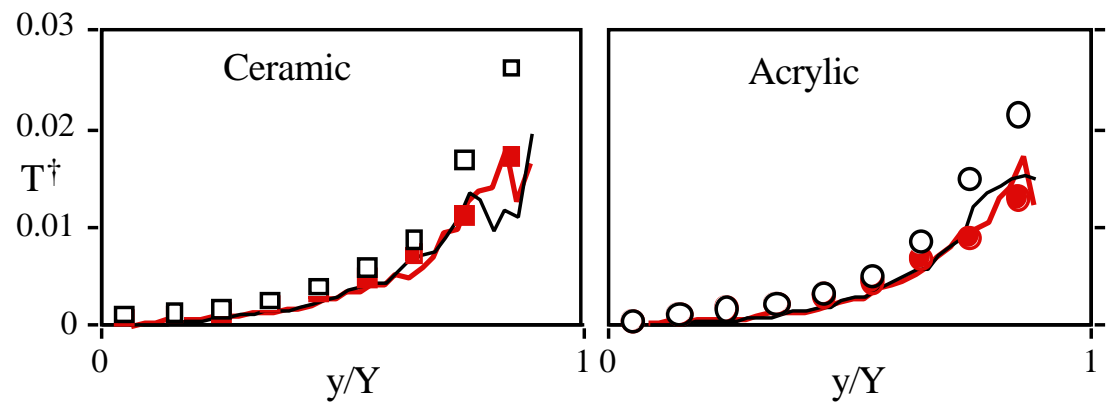

Figure 7. Transverse profiles of dimensionless second moments for the conditions of Test A. The symbols and lines are experimental measurements and predictions of the simulations, respectively. The open symbols and thin lines refer to the $\mathrm{x}$-direction, while the closed symbols and thick lines refer to the $\mathrm{y}$ direction.

Figures 6 to 8 compare experimental data with the predictions of the computer simulations for Test A. We observed similarly good agreement with the other two tests.

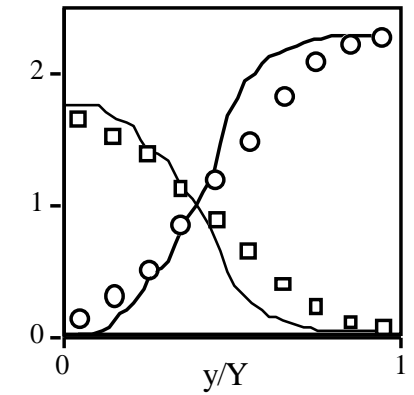

Figure 8. Transverse profiles of relative number fractions for the conditions of Test A. For symbols and lines, see Fig. 6.

\section{Conclusions}

The agreement between experiments and simulations demonstrated the utility of computer simulations as an equal partner to theory and physical experiment. In this event, fewer physical experiments need be done, and the simulations can be used with confidence as the basis of the theoretical modeling. Also, with advances in computing power, such simulation may eventually be employed to study the behavior of complete systems of technological importance that involve collisional granular flow. 


\section{M.Y. LOUGE, J.T. JENKINS, A. REEVES AND S. KEAST}

\section{References}

1. Savage, S. B. and Lun, C.K.K.: Particle size segregation in inclined chute flow of dry cohesionless granular solids, J. Fluid Mech. 189 (1988), 311-335.

2. Haff, P. K. and Werner, B. T.: Computer simulation of the mechanical sorting of grains, Powder Tech. 48 (1986), 239-245.

3. Rosato, A., Strandburg, K. J., Prinz F. and Swendsen, R. H.: Monte Carlo simulation of particulate matter segregation, Powder Tech. 49 (1986), 59-69.

4. Rosato, A., Strandburg, K. J., Prinz, F. and Swendsen, R. H.: Why the Brazil nuts are on top: size segregation of particulate matter by shaking, Phys. Rev. Lett. $\mathbf{5 8}$ (1987), 1038-1040.

5. Jenkins, J. T. and Askari, E.: Rapid granular shear flows driven by identical, bumpy, frictionless boundaries, in C. Thornton, (ed.), Powders and Grains 93, Balkema, Rotterdam, 1993, pp. 295-300.

6. Lorenz, A., Tuozzolo, C. and Louge, M.Y.: Measurements of impact properties of small, nearly spherical particles, Experimental Mechanics 37 (1997), 292-298.

7. Foerster, S., Louge, M. Y., Chang, H. and Allia, K.: Measurements of the Collision Properties of Small Spheres, Phys. Fluids 6 (1994), 1108-1115.

8. Hopkins, M. A. and Louge M.Y.: Inelastic Microstructure in Rapid Granular Flows of Smooth Disks, Phys. Fluids A 3 (1991), 47-57.

9. Walton, O.R.: Granular Solids Flow Project, Quarterly Report, UCID-20297-88-1, Lawrence Livermore National Laboratory, 1988.

\section{Acknowledgments}

This research was sponsored by NASA's Microgravity Science and Applications Division under contract NCC3-468. The specialty chain was donated by Morse Industrial, a division of Emerson Power Transmission Corporation.

The authors are indebted to Rowin Andruscavage, Gregory Aloe, Claudio Bazzichelli, Amelia Dudley, Patrick Florit, Joshua Freeh, Lance Hazer and Rami Sabanegh for measuring the impact properties in Table 1; to Fran McLeod for helping with the dc motor; to Edward Balaban, Taro Banno, Michael Chen, Michael Garon, Marie-Noëlle Pons and Christopher Salvestrini for developing a prototype of the vision algorithm; and to Gregory Aloe, Birgir Arnarson, Roshanak Hakimzadeh, Jeffrey Larko and Elaina McCartney for participating in the KC-135 flights.

We are equally indebted to the engineering team at the NASA-Lewis Research Center: Joe Balombin, Christopher Gallo, Frank Gati, Roshanak Hakimzadeh, Jeffrey Larko, Pamela Mellor, Emily Nelson, Enrique Ramé, Leon Rasberry, John Yaniec and Gary Wroten; and to the KC-135 flight crews and ground support team. 